\title{
Akute myeloische Leukämie
}

\section{Busulfan plus Fludarabin neuer Standard?}

Bei älteren Patienten mit akuter myeloischer Leukämie (AML) ist das myeloablative Konditionierungsregime Busulfan plus Fludarabin im Vergleich zur Kombination aus Busulfan plus Cyclophosphamid mit einer niedrigeren trans-

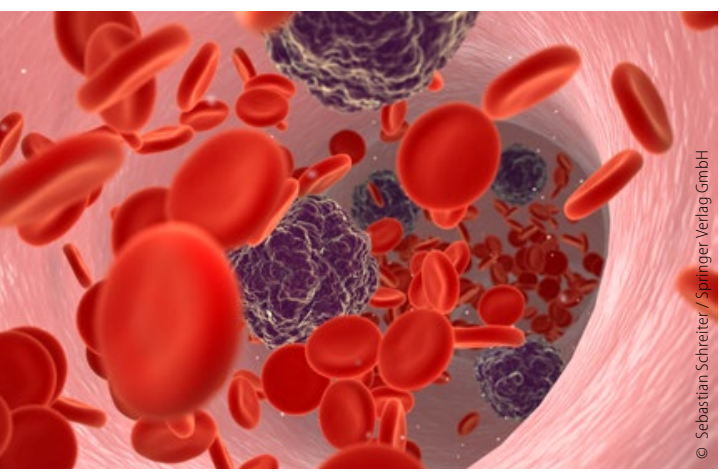

Überzahl an Myeloblasten im Blut eines AML-Patienten. plantatbedingten Mortalität und einer beträchtlichen antileukämischen Aktivität verbunden. Das zeigte eine Phase-IIIStudie. Das Regime sollte nach Meinung der Forscher der neue Behandlungsstandard sein.

An der multizentrischen, randomisieten, offenen Studie nahmen 25 Transplantationszentren in Italien und eines in Israel teil. Die auswertbaren Patienten $(\mathrm{n}=252)$ waren zwischen 40 und 65 Jahre alt, hatten einen ECOG-Performancestatus von weniger als 3 und befanden sich in vollständiger Remission.

Die Patienten in der Gruppe mit Busulfan/Cyclophosphamid-Therapie erhielten viermal täglich $0,8 \mathrm{mg} / \mathrm{kg}$ Busulfan in einer einstündigen Infusion an vier aufeinanderfolgenden Tagen. Zudem bekamen die Patienten $60 \mathrm{mg} / \mathrm{kg}$ Cyclophosphamid pro Tag jeweils an den Tagen -4 und -3 .
Die Patienten in der Busulfan/Fludarabin-Gruppe bekamen die gleiche Dosis Busulfan, allerdings an den Tagen -6 bis -3 ; zudem erhielten sie Fludarabin in einer Dosis von $40 \mathrm{mg} / \mathrm{m}^{2}$ pro Tag an den Tagen -6 bis -3 .

Die mittlere Nachbeobachtungsdauer lag bei 27,5 Monaten. Nach einem Jahr betrug die rezidivfreie Mortalität 17,2\% in der Busulfan/CyclophosphamidGruppe und 7,9\% in der Busulfan/Fludarabin-Gruppe $(\mathrm{p}=0,026)$.

Die häufigsten Nebenwirkungen vom Grad 3 oder mehr waren gastrointestinaler Natur (23\% unter Busulfan/Cyclophosphamid und $21 \%$ unter Busulfan/ Fludarabin). Zudem trat bei $17 \%$ bzw. $10 \%$ der Patienten mindestens eine Infektion auf.

Christina Berndt

Rambaldi A et al. Busulfan plus cyclophosphamide versus busulfan plus fludarabine as a preparative regimen for allogeneic haemopoietic stem-cell transplantation in patients with acute myeloid leukaemia: an open-label, multicentre, randomised, phase 3 trial. Lancet Oncol. 2015; 16(15):1525-36.

\section{Anti-VEGF-Inhibitoren bei mRCC \\ Wann droht ein Therapieabbruch?}

In der bisher größten Datensammlung zur Anti-VEGF-Therapie bei metastasiertem Nierenzellkarzinom (mRCC) waren Alter, glomeruläre Filtrationsrate, Metastasenanzahl und der Natriumspiegel unabhängige Risikofaktoren für toxizitätsbedingte Therapieabbrüche. Ausgewertet wurden Daten von 936 mRCC-Patienten, die in der Erstlinie eine gegen VEGF gerichtete Therapie erhalten hatten. Am häufigsten war Sunitinib (77\%) eingesetzt worden, gefolgt von Sorafenib (18,4\%), Bevacizumab (3,8\%) und Pazopanib (0,9\%).

Median betrug die Behandlungsdauer 7,1 Monate in der gesamten Studienpopulation und 4,4 Monate bei Patienten mit toxizitätsbedingten Therapieabbrüchen. 833 Patienten hatten die Therapie vorzeitig beendet, 23,8\% aufgrund von Toxizitäten, darunter Fatigue (4\%), Diarrhö (2\%) und Appetitmangel (2\%). Häufigste Gründe für Dosisreduktionen waren Fatigue

\section{kurz notiert}

\section{Tyrosinkinase trotz Inhibition weiterhin aktiv}

Die Tyrosinkinase 2 (Tyk2) ist eine Januskinase, die an der Entzündung, Karzinogenese und an der Abwehr von Infektionen beteiligt ist. Zwar kann die Hemmung der Enzymaktivität von Tyk2 das Krebswachstum stoppen, die Wirkung von Tyk2 als Immunzellaktivator bleibt jedoch erhalten [Prchal-Murphy M et al. Oncoimmunology. 2015;4(11):e1047579]. Aus therapeutischer Sicht ist dies vorteilhaft, denn Tyk2 trägt wesentlich zur Aktivierung der natürlichen Killerzellen bei, die an der Tumorbekämpfung beteiligt sind. In Mäusen, bei denen die Kinaseaktivität der Tyk2 ausgeschaltet wurde, war das Krebswachstum stark gehemmt. Dennoch behielten die natürlichen Killerzellen die Fähigkeit, Krebszellen zu bekämpfen. Das bedeutet, dass Kinaseinhibitoren für die Krebstherapie gut geeignet sind.
(12\%), Hand-Fuß-Syndrom (10\%) und Mukositis (7\%). Die kumulative 1-JahresInzidenz von toxizitätsbedingten Therapieabbrüchen lag bei $17 \%$ unter Sunitinib und bei $23 \%$ unter Sorafenib. Signifikante Prädiktoren für toxizitätsbedingte Therapieabbrüche waren nach der multivariaten Analyse: eine glomeruläre Filtrationsrate $<30 \mathrm{ml} / \mathrm{min} / 1,73 \mathrm{~m}^{2}$ (Hazard Ratio [HR] $2,2 ; \mathrm{p}=0,122)$, eine einzelne Metastasenlokalisation (HR 1,51; $\mathrm{p}=0,096$ ), ein $\mathrm{Na}$ triumspiegel $<135 \mathrm{mmol} / \mathrm{l}$ (HR 1,81; $\mathrm{p}=$ 0,0056) und ein Alter $\geq 60$ Jahre zu Studienbeginn (HR 1,79; $p=0,0022$ ).

Basierend auf der Zahl vorhandener Risikofaktoren wurden die Patienten in Risikogruppen eingeteilt. Ein niedriges Risiko lag bei keinem oder einem Risikofaktor vor, ein mittleres Risiko bei zwei Risikofaktoren und ein hohes Risiko bei drei oder vier Risikofaktoren.

Judith Neumaier

Kaymakcalan MD et al. Risk Factors and Model for Predicting Toxicity-Related Treatment Discontinuation in Patients With Metastatic Renal Cell Carcinoma Treated With Vascular Endothelial Growth Factor-Targeted Therapy: Results From the International Metastatic Renal Cell Carcinoma Database Consortium. Cancer. 2016;122(3):411-9. 